\title{
NTIC et co-développement
}

New information and communication technologies and co-development

Nuevas tecnologías de información y comunicación y co-desarrollo

Jean-Yves Babonneau, Alain Caristan, Sandra Coulombier et Vincent

Tournardre

\section{CpenEdition}

\section{Journals}

Édition électronique

URL : http://journals.openedition.org/ries/2737

DOI : $10.4000 /$ ries. 2737

ISSN : 2261-4265

Éditeur

Centre international d'études pédagogiques

Édition imprimée

Date de publication : 23 septembre 1999

Pagination : 67-74

ISSN : $1254-4590$

Référence électronique

Jean-Yves Babonneau, Alain Caristan, Sandra Coulombier et Vincent Tournardre, «NTIC et codéveloppement », Revue internationale d'éducation de Sèvres [En ligne], 23 | 1999, mis en ligne le 23 septembre 1999, consulté le 19 avril 2019. URL : http://journals.openedition.org/ries/2737 ; DOI : $10.4000 /$ ries. 2737 


\title{
NTIC et \\ co-développement
}

\author{
Jean-Yves Babonneau, \\ Alain Caristan, \\ Sandra Coulombier, \\ Vincent Tournardre
}

\section{New information and communication technologies and co-development}

The development of telematic networks facilitate worldwide exchanges and place industrialised countries and underprivileged countries on equal terms : it gives credit to on-line products, wherever they come from. This new process of co-development implies in-depth knowledge of hardware and systems. This article shows the part played by international organisations in this field, in particular French speaking organisations.

\section{Nuevas tecnologías de información y comunicación y co-desarrollo}

El desarrollo de redes telemáticas facilita los intercambios a nivel mundial y puede poner a los países industrializados y países desfavorecidos en el mismo nivel : valoriza los productos en línea, sea cual fuere el lugar de concepción. Ese nuevo proceso de co-desarrollo implica el dominio de materiales y dispositivos. El artículo muestra la acción de las organizaciones internacionales, en particular las organizaciones francófonas.

Le développement de ce qu'il est désormais convenu d'appeler les nouvelles technologies de l'information et de la communication (NTIC) permet aujourd'hui de favoriser la connaissance, d'encourager la coopération entre nations et l'échange international ${ }^{1}$. Il conduit de grands organismes ${ }^{2}$ à adopter

1 Certaines parties de ce document sont extraites de : Co-développement et inforoutes de la Commission française pour l'UNESCO, rédigé par Jean-Yves Babonneau, juin 1996 ; Forum initiatives de l'AUPELF-UREF et de l'Internet Society, rédigé par Alain Caristan, novembre 1997.

2 UNESCO (Organisation des Nations unies pour l'éducation, la science et la culture), AFNIC (Association française pour le nommage internet en coopération), AUF (Agence universitaire de la francophonie), l'Agence de la francophonie (ex-ACCT), INRIA (Institut national de recherche en informatique et en automatique), ISOC (Internet Society). 
de nouveaux modes d'action, car il est aujourd'hui nécessaire d'œuvrer pour un développement équilibré de ces nouvelles technologies. Il importe de contribuer à la satisfaction des besoins des différents partenaires, en favorisant la mise en valeur des patrimoines mais aussi la libre circulation de l'information à un niveau local et international, en permettant de rompre l'isolement des acteurs et potentiellement, de tous les individus, et plus ambitieusement, de donner aux pays aujourd'hui défavorisés, les moyens et les concours qui leur permettent, dans le cadre d'une politique de co-développement, de participer au même titre que les «pays du Nord » à la construction d'une nouvelle société.

Le co-développement, développement de tous les pays en parallèle, se fonde sur des transferts de compétences des pays industrialisés vers les pays en émergence et sur la logistique des systèmes d'information accessibles par les inforoutes.

\section{Inforoutes et co-développement: fondements}

Au lieu de s'inquiéter du déplacement de certaines activités économiques dans les pays à faible niveau de vie, le co-développement aide aujourd'hui ces derniers à sortir de leur position majoritaire de consommateurs pour leur permettre de devenir des fournisseurs de biens valorisés sur le marché international. Pour ce faire, il importe de contribuer à moyen et long terme à former dans ces pays des générations d'entrepreneurs, à développer l'offre locale ouverte aux pays industrialisés, à faciliter l'accès aux informations à égalité de chances et à élargir les moyens d'attirer les entreprises.

Face à une dérive mercantile, les cultures peuvent se développer par la richesse des valeurs qu'elles prônent et par l'exemple qu'elles donnent de leur mise en œuvre.

Par ailleurs, une logistique de communication adaptée aux télé-activités (réseaux, internet, etc.) rompt l'isolement des personnes qui, revenant ou provenant des pays industrialisés, assurent les transferts de savoirs et de contenus culturels et participent au développement local.

Pour les pays industrialisés, l'objectif du co-développement est d'apporter la pleine activité des systèmes de production en favorisant la solvabilité des clients qui peuvent alors financer leurs besoins en équipements. On s'attend à un développement des activités de formation en raison de l'effort considérable de transfert de connaissances qui est nécessaire. De nombreux projets pilotes, présentés dans ce document, ont déjà été menés dans cette voie avec succès.

Le co-développement, lié au multilinguisme et porteur d'un idéal humaniste et riche en espoirs sociaux, notamment pour la résorption du chômage, suscite l'intérêt, tant par son contenu culturel que pour ses objectifs économiques. 


\section{Les inforoutes}

Pour maîtriser son avenir, l'homme a modélisé son environnement et accumulé son expérience dans un espace virtuel de données dont le volume s'accroît avec sa connaissance. Le système informatique gère aujourd'hui ces données au sein d'une architecture distribuée dont le cœur est un réseau qui véhicule les flux engendrés par les échanges de données. Autour du système informatique, il y a non pas un homme isolé mais une organisation humaine, hiérarchisée, cloisonnée et cependant coopérante.

Une approche globale (organisation humaine/système informatique) conduit à concevoir la notion de système d'information comme une logistique de coopération, nécessairement ouverte à l'échelle planétaire. La transparence de la localisation des traitements, des données et de l'organisation doit permettre d'atteindre un objectif majeur du système d'information : la maîtrise globale des activités de collaboration, d'interaction entre individus au sein d'une même organisation comme entre organisations, quelle que soit leur localisation.

\section{Le marché des technologies de l'information}

$\mathrm{Au}$ cours de ces dernières années une forte accélération s'est produite dans le domaine des technologies de l'information due à la pénétration du marché grand public. Les conséquences en sont considérables : prix de fabrication de masse, performances des microprocesseurs, transparence de la complexité technologique, développement des infrastructures de réseau et des services hypermédias répartis.

Ainsi, la distribution des équipements et l'accès à l'information partout dans le monde deviennent aujourd'hui possibles : d'une part, par la mise à disposition de ces équipements auprès du grand public dans le monde industrialisé, qui conduit à un abaissement de leurs coûts ; d'autre part, par les avancées technologiques qui renforcent les possibilités de diffusion de ces outils.

\section{La révolution multimédia}

Depuis peu la numérisation des informations et leur circulation sur des réseaux planétaires modifient radicalement nos modes d'être, d'agir et d'entretenir nos relations les uns avec les autres. L'apparition et l'utilisation croissante d'outils de formation pédagogiques, tout comme les capacités de mémorisation des informations offertes par ces outils ne sont que deux exemples de la puissance des instruments multimédias. 
La prise en compte de cette dimension multimédia par le système informatique permet également d'accélérer la compréhension et d'améliorer la collaboration entre individus.

\section{Normes, standards ouverts et internet}

L'importance du système d'information dans les échanges entre les hommes et sa dimension planétaire imposent d'appuyer son développement sur des normes et des standards ouverts. L'internet constitue un excellent exemple de standard du domaine public satisfaisant l'objectif de gestion décentralisée et disponible sur toutes les plate-formes.

L'internet est aujourd'hui un véritable système d'information planétaire couvrant la quasi totalité des pays. Le nombre de machines connectées à l'internet double tous les dix mois et le nombre des serveurs d'information, tel que le Web, croît de façon exponentielle. Cette évolution, qui bénéficiait encore récemment aux pays industrialisés, commence à profiter aux pays en émergence, car ce réseau et ses services constituent un instrument majeur de développement dans les pays à industrialiser.

L'architecture de l'internet permet ce développement car elle met en œuvre un processus totalement décentralisé, fondé sur la collaboration et le partage, en offrant une grande souplesse d'adaptation à toutes sortes de supports physiques de connexion. L'internet autorise ainsi une collaboration aisée entre des individus très dispersés sur la surface du globe et apparaît donc comme remarquablement adapté à une politique de co-développement.

\section{Le co-développement en action}

De grands organismes internationaux francophones s'intéressent aujourd'hui de près au co-développement qui semble être un moyen efficace, et peu coûteux, d'aide au développement des pays en émergence car il s'appuie sur les NTIC.

Les principales organisations présentes sur le marché du co-développement sont l'UNESCO, l'AUF, l'Agence de la francophonie, l'AFNIC, l'ISOC. Elles apportent leur aide au co-développement en organisant et en finançant des ateliers de formation de formateurs aux technologies de l'information (FFTI), en envoyant des formateurs (ingénieurs, chercheurs, pédagogues) dans les pays en émergence pour transférer les compétences d'un pays à l'autre, ou en produisant des supports de cours ou des outils de formation afin de les distribuer au plus grand nombre. 


\section{Les ateliers INET ${ }^{3}$-FFTI}

La formation est l'un des secteurs qui s'intéressent aux NTIC non seulement pour les aspects de contenus mais aussi pour leur apport dans les méthodes d'apprentissage.

Les organisations citées précédemment participent à ce processus en opérant sur les réseaux de recherche et en animant des activités de recherche et de développement dans le monde francophone. Une action est en cours pour définir et mettre en ouvre une politique régionale de formation aux NTIC dans les pays francophones. Il s'agit d'ateliers de FFTI.

A travers la formation de spécialistes, trois objectifs sont visés :

- démontrer le potentiel des NTIC et aider à leur appropriation régionale ;

- atteindre la masse critique de spécialistes techniques dans un secteur d'activité donné, pour une région donnée ;

- augmenter le potentiel des enseignants, en apportant une méthodologie et des outils de formation réutilisables par les stagiaires appelés à devenir formateurs à leur tour.

Le principe de l'atelier est la formation, par la pratique, de techniciens dans un domaine de mise en œuvre des NTIC. La formule a été lancée en 1996 à Montréal par les ateliers de l'internet de l'ISOC en partie francophones. Par la suite, pour plus d'efficacité, des ateliers se sont tenus à Tunis en mai 1997 et à Genève en juillet 1998 dans le cadre de RAITNET ${ }^{4}$.

L'AUF qui compte renforcer cette action dans le contexte du co-développement dans la francophonie a, pour sa part, organisé des ateliers INET-FFTI francophones au Vietnam (octobre 1997), à Madagascar (juin 1998), en Haïti (novembre 1998), au Bénin (février 1999) et au Liban (avril 1999) 5 .

Par l'action de formation, les ateliers régionaux FFTI contribuent à l'animation des groupes culturels, voire économiques, régionaux ainsi qu'au renforcement du tissu relationnel et technique régional.

En intégrant des stagiaires issus d'autres régions, ils contribuent à accélérer le développement de régions démunies de techniciens, à élaborer les formations à venir dans ces régions, à préparer ou renforcer les actions de coopération à l'échelle mondiale sur les inforoutes.

Les ateliers FFTI doivent répondre à l'attente du monde francophone pour l'aider à maîtriser les NTIC. Il est nécessaire pour cela de dépasser le strict

3 Inet Informatique.

4 Ces ateliers ont été organisés par l'IRSIT (Institut régional des sciences informatiques et des télécommunications), I'ATI (Agence tunisienne de l'information) et I'INRIA. IIs ont reçu le soutien du programme PII (programme intergouvernemental d'informatique) de l'UNESCO.

5 Voir article de Didier Oillo. 
cadre technique pour s'inscrire dans un plus vaste plan de développement durable des réseaux francophones. Ces formations attachent autant d'importance au fond qu'à la forme et se doivent d'expliquer le rôle et la mission des formateurs pour créer un véritable climat de coopération technique et culturelle.

Les ateliers de formation INET-FFTI ont une utilité certaine et un rôle à la fois pédagogique et moteur en matière de développement des inforoutes. Ils montrent l'intérêt et la nécessité de poursuivre dans la voie du co-développement des NTIC avec les pays en émergence. L'approche régionale, associée à l'identification culturelle francophone, crée les conditions favorables à des échanges techniques durables.

L'expertise technologique et organisationnelle, mise ainsi au service du transfert des connaissances et à l'acquisition du métier d'internaute, contribue à affirmer et à renforcer une participation active de la francophonie au développement des contenus sur l'internet. Le rôle de la francophonie et sa force apparaissent ici dans l'émergence de réseaux et de projets durables sur les inforoutes. Ces formations participent à la dissémination des technologies de l'internet introduites par l'ISOC. Elles entendent aller plus loin en prolongeant la coopération entre les participants.

Ce constat positif ne doit cependant pas faire oublier que la formule n'en est qu'à ses débuts. Elle est, aujourd'hui, loin d'avoir abouti. On en est seulement à la phase d'incursion dans un domaine complexe qui combine évolution des méthodes de formation et mutation des sociétés vers le marché de l'information.

Des projets naissants, comme l'université virtuelle de l'AUF qui veut doter les acteurs francophones de la recherche et du développement de moyens modernes pour produire, échanger, transformer l'information à des fins de développement social et économique, trouvent ici une manifestation concrète de leur potentiel.

Ces ateliers permettent de quantifier les objectifs des programmes de formation FFTI. Ils s'inscrivent pleinement dans les actions de co-développement des NTIC dans les pays en émergence. Ils créent les conditions de rendezvous réguliers qui doivent, à terme, assurer une présence permanente sur l'internet des différentes cultures qui s'y côtoient et interopèrent. Ils renforcent le dispositif qui tend à rapprocher les hommes et les cultures par le réseau. Ils apportent l'autonomie de production et d'échange par les moyens les plus modernes, tout en permettant de conserver une identité culturelle et d'ouvrir de nouveaux marchés.

\section{Une nouvelle donne de formation}

Trois principaux modes de formation sont aujourd'hui complémentaires :

- la formation présentielle, la seule il y a encore peu de temps, est la for- 
mation où un formateur (enseignant) se trouve physiquement dans la même pièce que le ou les apprenants (élèves, stagiaires) ;

- la formation à distance, qui a fait son apparition il y a quelques années grâce aux nouvelles technologies comme la vidéoconférence sur l'internet (mise au point en France par l'équipe d'Alain Caristan, chercheur à l'INRIA) : ce mode de formation fait appel, comme la formation présentielle, à un formateur et à des enseignants, qui ne se trouvent pas physiquement dans une même pièce, mais communiquent entre eux grâce à des supports multimédias ;

- l'autoformation, qui se concrétise aujourd'hui par des outils pédagogiques multimédias mis au point par des professionnels, permet à l'apprenant de se former à son rythme, sans contrainte de temps ou de lieu. Elle permet de démultiplier la formation pour la mettre sur le marché de masse.

Les NTIC redéfinissent complètement les modes d'apprentissage de chacun et, plus particulièrement, le rapport enseignant/enseignés. Avant, les enseignés se trouvaient sur un cercle au milieu duquel prenait place l'enseignant. Aujourd'hui, l'enseignant et les enseignés se trouvent sur le même cercle, et le rôle de l'enseignant diffère en ce qu'il sert de conseiller, et non plus de formateur. En effet, sur ce même cercle, les enseignés peuvent eux-mêmes répondre aux questions posées à distance par les autres enseignés. L'enseignant quant à lui accompagne les échanges et les dynamise.

Chaque cercle peut être relié à un autre par des connexions de réseaux. L'information est ainsi démultipliée, et ce nouveau mode de formation a l'avantage d'apporter à chaque enseigné une même formation, enrichie par les aspects culturels et linguistiques des enseignés de tous les autres pays.

Concrètement, l'AFNIC a mis au point deux outils d'autoformation dans le but de déployer l'internet et de transférer les compétences au plus grand nombre, rapidement, et à moindre coût ${ }^{6}$. Le premier outil d'autoformation, «Initi@tion à l'Internet », forme l'apprenant à l'internet et ses différents services: navigation sur le web, envoi et réception de messages électroniques, transfert de fichier, création d'une page web, etc. L'outil d'autoformation«A ch@cun son site web » est un outil d'aide à la création de sites web. Il propose un cycle en quatre phases : concevoir, réaliser, installer et administrer son site web.

Ces deux outils permettent à l'apprenant de s'autoformer sans appréhension, car ils proposent des animations interactives pédagogiques qui guident l'apprenant dans sa formation, des fiches web qui répondent à des questions pratiques et des modules d'auto-évaluation. Conçus avec une démarche pédagogique validée par des enseignants et des formateurs, ils répondent à une véritable demande du public.

6 Pour plus d'informations, consultez le site web de l'AFNIC : « http://www.afnic.asso.fr ». 
Les nouvelles technologies de l'information contribuent indéniablement à faire évoluer en profondeur nos sociétés. Mais ces technologies constituent avant tout des outils au service de la cause que d'aucuns voudront bien leur confier.

Face à la logique, à court terme, liée à la crise économique mondiale et dans laquelle les États industrialisés tendent à s'enfermer, de grands organismes trouvent une mission conforme à leur vocation éthique et à leur mode de fonctionnement : la mise en œuvre d'une politique sur le moyen et le long terme, au service de tous et de chacun, dans le respect et la mise en valeur de la diversité culturelle et linguistique de ce monde. 